\title{
Efficient Bandwidth and Power Allocation Algorithms for Multiuser MIMO-OFDM Systems
}

\author{
Jin SHU, Wei GUO \\ The Wireless Information Network Laboratory, University of Science and Technology of China, Hefei, China \\ Email:\{shujin,wei\}@mail.ustc.edu.cn \\ Received December 24, 2008; revised March 5, 2009; accepted May 26, 2009
}

\begin{abstract}
This paper studies the problem of finding an effective subcarrier and power allocation strategy for downlink communication to multiple users in a MIMO-OFDM system with zero-forcing beamforming. The problem of minimizing total power consumption with constraint on transmission rate for users is formulated. The problem of joint allocation is divided into two stages. In the first stage, the number of subcarriers that each user will get is determined based on the users' average signal-to-noise ratio. In the second stage, it finds the best assignment of subcarriers to users. The optimal method is a complex combinatorial problem which can only be assuredly solved through an Exhaustive Search (ES). Since the ES method has high computational complexity, the normalized user selection algorithm and the simplified-normalized user selection algorithm are proposed to reduce the computational complexity. Simulation results show that the proposed low complexity algorithms offer better performance compared with an existing algorithm.
\end{abstract}

Keywords: Multiuser, MIMO-OFDM, Adaptive Resource Allocation, QoS

\section{Introduction}

The multiuser MIMO-OFDM system has great potential of providing enormous capacity due to its integrated space-frequency diversity and multiuser diversity. Assuming knowledge of channel state information (CSI) is available at the transmitter, the performance can be further improved through the adaptive resource allocation. For the OFDMA systems with single antenna, several resource allocation methods were proposed in [1-3] to minimize the total transmit power given QoS by utilizing the multiuser diversity in frequency domain. $[4,5]$ investigated the SDMA-OFDM system in an environment with multi-antenna equipped at the base station. [4] proposed an optimal lagrangian iteration method to maximize the system throughput under the total power constraint. Because the optimal scheme is complicated, a greedy algorithm was proposed to reduce the complexity in [5].

Considering a multiuser MIMO-OFDM system with downlink beamforming, it is assumed that the base station can acquire perfect CSI, [7] employed the SUS (Semi-orthogonal User Selection) algorithm proposed in

"Manuscript received, 2008. This work was supported by the National Basic Reseasch Program of China (973 program), 2007CB310602.
[6] to minimize the total transmit power satisfying the QoS of users. But in [7] the size of SDMA group was fixed, therefore, the orthogonality of channels of users in a group was not well guaranteed.

In order to guarantee the orthogonality of channels of users in a group, we propose the Normalized User Selection (NUS) algorithm. In NUS algorithm, each user group is regards as a virtual user, the number of users in a group is normalized to unitary, and then the resource allocation schemes for OFDMA can be employed. The NUS scheme has to traverse all the user groups on each subcarrier, obviously, the computation complexity is large when there are lots of users. In order to further reduce the complexity, the S-NUS algorithm (Simplified-NUS) is proposed. On each subcarrier, a user with the channel which has the largest magnitude and lowest correlation with the other already selected users is selected. The way to calculate the spatial correlation among users is employed as in [6]. When the number of users is huge, the S-NUS algorithm can greatly reduce the complexity. In our proposed algorithms, the number of users on each subcarrier is not fixed but depends on the spatial correlation of users. Since the number of users on each subcarrier is not a constant, it is hard to count the number of subcarriers for each user. In order to count the number of subcarriers easily, we pull-in the statistical 
weights of subcarriers. With the statistical weight, the Bandwidth Assignment Based on SNR (BABS) algorithm proposed in [2] can be applied to determine the number of subcarriers for each user. Simulation results show that both of the NUS and S-NUS algorithms can achieve better performance than the algorithm in [7]. Compared to NUS algorithm, the S-NUS algorithm has lower complexity but with little performance loss, and is a better choice.

This paper is organized as follows. Section 2 presents the system model and the formulation of the problem. Section 3 introduces two sub-optimal resource allocation algorithms. Section 4 shows the simulation results. Finally, Section 5 concludes the paper.

Notation: We use $(.)^{T}$ stands for the transpose of a matrix (vector), $(.)^{\dagger}$ stands for the pseudo-inverse of a matrix, $(.)^{*}$ stands for the conjugate transpose of a matrix, $|\mathrm{A}|$ denotes the size of the set $A,[\mathrm{~A}]_{k, k}$ represents the $k$ th diagonal element of $A$.

\section{System Model}

\subsection{Channel Model and Transmit Structure}

We consider a downlink MIMO-OFDM system with a base station supporting data traffic to $K$ user terminals. The base station is equipped with $M$ transmit antennas and each user terminal has a single receive antenna. We assume that $K \geqslant M$.The frequency band is divided into $N$ subcarriers. It is considered that the channel matrix dose not vary during the coherence interval of $T$.The received signal of user $k$ on subcarrier $n$ can be represented as

$$
y_{k, n}=\mathbf{h}_{k, n} \mathbf{x}_{n}+z_{k, n}
$$

Where $\mathbf{h}_{k, n} \in \mathbf{C}^{1 \times M}$ is the channel gain matrix of user $k$ and entries of $\mathbf{h}_{k, n}$ are assumed to be identically independent distributed with zero mean and unit variance, $x_{n} \in \mathbf{C}^{M \times 1}$ is the transmit symbol from the base station antennas, $z_{k, n}$ is complex Gaussian noise with zero mean and unit variance of user $k$.

At the transmitter, we employ the zero-forcing beamforming (ZFBF) transmit strategy. In ZFBF, the transmitter selects an active user set $S_{n} \subset\{1, \ldots K\}$ of size $\left|S_{n}\right| \leq M$ to which data will be transmitted. The data symbol $S_{j, n}$ is multiplied by the beamforming vector $\mathbf{w}_{\mathrm{j}, \mathbf{n}}$ as follows

$$
\mathbf{x}_{n}=\sum_{j \in S_{n}} \sqrt{P_{j, n}} \mathbf{w}_{j, n} S_{j, n}
$$

Then the received signal (1) becomes

$$
y_{k, n}=\mathbf{h}_{k, n} \sum_{j \in S_{n}} \sqrt{P_{j, n}} \mathbf{w}_{j, n} s_{j, n}+z_{k, n}
$$

In [6], the beamforming vector is selected to satisfy the zero-interference condition $\mathbf{h}_{k, n} \mathbf{w}_{j, n}=0$, for $j \neq k$. Denote $\mathbf{H}_{n}\left(S_{n}\right)$ and $\mathbf{W}_{n}\left(S_{n}\right)$ be the corresponding submatrices of $\mathbf{H}_{\mathbf{n}}=\left[\mathbf{h}_{1, n}^{T}, \ldots, \mathbf{h}_{K, n}^{T}\right]^{T}, \quad \mathbf{W}_{n}=\left[\mathbf{w}_{1, n, \ldots} \mathbf{w}_{K, n}\right]$, respectively.

The beamforming matrix $\mathbf{W}_{n}\left(S_{n}\right)$ can be simply obtained using pseudo inverse of $\mathbf{H}_{n}\left(S_{n}\right)$ as follows:

$$
\mathbf{W}_{n}\left(S_{n}\right)=\mathbf{H}_{n}\left(S_{n}\right)^{\dagger}=\mathbf{H}_{n}\left(S_{n}\right)^{*}\left(\mathbf{H}_{n}\left(S_{n}\right) \mathbf{H}_{n}\left(S_{n}\right)^{*}\right)^{-1}
$$

\subsection{Problem Formulation}

Since ZFBF can transmit $M$ spatial sub-stream simultaneously, maximum $M$ users can be allocated by ZFBF in each subcarrier. Let $\rho_{k, n}$ indicate whether the user $k$ is chosen on subcarrier $n$, denote $C_{\mathrm{k}, \mathrm{n}}$ indicate that user $k$ can transmit $c$ bits on subcarrier $n, \rho_{k, n}=1$ if $C_{k, n} \neq 0$, $\rho_{k, n}=0$ if $C_{k, n}=0$ 。

$$
\begin{aligned}
& \sum_{k=1}^{K} \rho_{k, n} \leq M, n=1, \ldots N . \\
& \sum_{n=1}^{N} c_{k, n}=R_{k}, k=1, \ldots K
\end{aligned}
$$

Where $R_{k}$ stands for the number of bits user $k$ want to transmit every symbol. Constraint (5) means at most $M$ users could be assigned to one subcarrier, constraint (6) means $R_{k}$ bits should be transmitted per symbol for user $k$.

The optimization problem can be formulated in the sense of the total transmit power satisfying (5), (6) as follows.

$$
\min _{\rho_{k, n}, c_{k, n}} \sum_{n=1}^{N} \sum_{k=1}^{K} \frac{f_{k}\left(c_{k, n}\right)}{\gamma_{k, n}} \rho_{k, n}
$$

subject to (5), (6)

Where

$$
\gamma_{k, n}=\frac{1}{\left[\left(\mathbf{H}\left(S_{n}\right) \mathbf{H}\left(S_{n}\right)^{*}\right)^{-1}\right]_{k, k}}, k \in S_{n}
$$

is the effective channel gain on subcarrier $n$ for user $k$. $f_{k}(c)$ stands for the required transmit power to transmit $c$ bits when channel gain is unity. When uncoded $2^{c}$ - ary QAM is employed, the required transmit power 
can be tightly approximated as [8]:

$$
f_{k}(c)=\frac{N_{0}\left(1-2^{c}\right) \log \left(5 B E R_{k}\right)}{1.6}
$$

Where $B E R_{k}$ is the bit error rate of user $k, N_{0}$ is the variance of Gaussian white noise and is assumed to be unitary in this paper.

\section{Subcarrier and Bit Allocation}

The solution of optimization problem (7) can be separated into three stages. In the first stage, the number of required subcarriers for each user is roughly determined based on target rate and the average channel gain of each user. In the second stage, allocate subcarriers to each user according to the number of subcarriers obtained in the first stage. In the third stage, bit allocation for assigned subcarriers to each user is performed. For each user, a greedy algorithm for single user is employed to allocate bits as in [1].

\subsection{Resource Allocation}

In a wireless environment, the channel state of some users will be inferior to others'; these users tend to need more transmit power. As shown in [1-3], more subcarriers should be assigned to these users with lower average channel gain to satisfy the rate constraint of these users. Since the number of users is not stationary for the MIMO-OFDM systems with ZFBF on each subcarrier, it is hard to count the number of subcarriers for each user. If the number of subcarriers for each user is added up one by one, the result is that the number of subcarriers is not a constant, so it is hard to determine whether the number of subcarriers for each user is satisfied. We assume that only one user transmit data on a subcarrier, the rate of the user is $r$, when there are two user transmit data on this subcarrier ,the rate of each users is approximated as $r / 2$, so, each user is regarded as to be assigned half of a subcarrier. And so forth, when there are three and four uses transmit data on a subcarrier, each user is regarded as to be assigned one third and one fourth of a subcarrier. Therefore, we pulls-in the statistical weights of subcarriers. Let $S_{n} \subset\{1, \ldots K\}$ be a subset of user indexes on subcarrier $n$ and $M_{n}=\left|S_{n}\right|$. When the user $k$ is selected, the number of subcarriers of it adds $1 / M_{n}$. In accordance with this, the sum of the number of subcarriers for all users is exactly $N$. In this way, the resource allocation algorithm for OFDMA can be employed for the MIMO-OFDM system considered in this paper. Assuming each user $k$ experiences of the identical channel gain for each subcarrier

$$
\gamma_{k}=\frac{1}{N} \sum_{n=1}^{N}\left\|\mathbf{h}_{k, n}\right\|^{2},
$$

the total number of subcarriers for user $k$ is $n_{k}$. When the channel gain is identical for each user on all subcarriers, the optimization of (7) is modified to find $n_{k}, k=1, \ldots, K$. The Bandwidth Assignment Based on SNR (BABS) algorithm proposed in [2] can be applied to find the solution of the above problem.

\subsection{Subcarrier Assignment Algorithm}

Once the number of subcarriers to each user is determined, the next step is to assign the specific subcarriers to each user. The original problem (7) is modified as the problem to find $\rho_{k, n}$.

$$
\min _{\rho_{k, n}} \sum_{n=1}^{N} \sum_{k=1}^{K} f_{k}\left(\frac{R_{k}}{n_{k}}\right) \frac{\rho_{k, n}}{\gamma_{k, n}}
$$

Subject to

$$
\begin{aligned}
& \sum_{k=1}^{K} \rho_{k, n} \leq M, n=1, \ldots N \\
& \sum_{n=1}^{N} \rho_{k, n} \leq M n_{k}
\end{aligned}
$$

In order to solve the above problem, two subcarrier assignment algorithms (NUS and S-NUS) are proposed in this paper.

\section{Algorithm 1. Normalized User Selection Algorithm (NUS)}

In OFDMA systems shown in [1-3], the subcarriers are assigned to the users with the largest channel gain to maximize the total throughout or minimize the total transmit power. Since in a multi-user MIMO-OFDM system with ZFBF, the effective channel gain depends on the orthogonality of channels of the user set assigned to a subcarrier, it is quite complicated to assign the subcarriers. In order to minimize the total transmit power, it is efficient to assign a user with the channel which has the largest magnitude and lowest correlation with the other already users assigned on a subcarrier. In [7], the number of users assigned simultaneously on each subcarrier is fixed as $M$. But it is difficult to select $M$ users with the channel which is low correlation with other already selected users while the number of total users is not large enough. Therefore, assign $M$ users simultaneously in a subcarrier is not good enough. In order to guarantee the orthogonality of the channels of users in a user set, we propose the NUS algorithm the user set of a subcarrier is regard as a virtual user by the proposed NUS algorithm. In NUS algorithm, the number of users in a user set is normalized to unitary; the best virtual user is selected on each subcarrier just the same as in OFDMA. Denote $\varphi_{n, p}(1 \leq p \leq P)$ be the $p$ th candidate user set on subcarrier $n, \varphi_{n, p} \subset\{1, \cdots K\},\left|\varphi_{n, p}\right| \leq M$, 


$$
P=\sum_{l=1}^{M}\left(\begin{array}{l}
K \\
l
\end{array}\right),\left(\begin{array}{l}
K \\
l
\end{array}\right)=\frac{K !}{l !(K-l) !} \text {. }
$$

The subcarrier assignment algorithm is shown as follow.

Step 1. Initialization

$$
\begin{aligned}
& U=\{1, \cdots, N\} ; T_{n}=\{1, \cdots, K\}, n=1, \cdots, N \\
& S_{n}^{0}=\varnothing, \rho_{k, n}=0, \forall k, n \\
& R_{k, a v e}=R_{k} / n_{k}, k=1, \cdots, K
\end{aligned}
$$

Step 2. Select the subcarrier

$$
\begin{aligned}
& \hat{n}=\arg \min _{\substack{n \in U \\
k \in T_{n}}} f_{k}\left(R_{k, a v e}\right) /\left\|\mathbf{h}_{k, n}\right\|^{2} ; U=U-\{\hat{n}\} \\
& \varphi_{\hat{n}, p} \leftarrow T_{\hat{n}}, \gamma_{\hat{n}, p, j} \leftarrow \varphi_{\hat{n}, p}, j \in \varphi_{\hat{n}, p}, p \in\{1, \cdots, P\}
\end{aligned}
$$

Step 3. Select the optimal user set

$$
\begin{aligned}
& r_{\hat{n}, p}=\sum_{j=1}^{M_{p}} f_{k}\left(R_{k, a v e} / M_{\hat{n}, p}\right) / \gamma_{\hat{n}, p, j}, j \in \varphi_{\hat{n}, p}, M_{\hat{n}, p}=\left|\varphi_{\hat{n}, p}\right| \\
& \hat{p}=\arg \min r_{\hat{n}, p}, p=1,2, \cdots, P \\
& \forall k \in \varphi_{\hat{n}, \hat{p}}, S_{\hat{n}}^{0}=S_{\hat{n}}^{0} \cup\{k\}, \rho_{k, \hat{n}}=1,
\end{aligned}
$$

Step 4. Count the number of subcarriers

$$
\begin{aligned}
& k \in S_{\hat{n}}^{0}, n_{k}=n_{k}-1 / M_{\hat{n}, p} \text {, if } n_{k} \leq 0, T_{n}=T_{n}-k, \forall n \\
& U=\Phi, \text { finish else go Step } 2
\end{aligned}
$$

In Step $1, T_{n}$ is the candidate user set of $n$th subcarrier, $\mathrm{U}$ is the candidate subcarrier set, $S_{n}^{0}$ is the selected user set of $n$th subcarrier, $R_{k}$ is the average bits user $k$ transmit each symbol, $n_{k}$ is the subcarriers user $k$ own determined by BABS, therefore, $R_{k, \text { ave }}$ is the average bits in the subcarriers for user $k$.

In Step 2, select the subcarrier $\check{n}$ with the minimum transmit power among users, each subcarrier is selected only once. $P$ is the total number of candidate user sets, $\varphi_{\check{n}, p}$ is the $P$ th user set of subcarrier $\check{n}, \gamma_{\check{n}, p, j}$ is the effective channel gain of user sets.

In Step 3, select the optimal user set based on the criterion: for the $P$ th user set, $M_{\check{n}, p}=\left|\varphi_{\check{n}, p}\right|$, after normalizing the number of users, each user is equivalent to $1 / M_{\check{n}, p}$ of a user. Therefore, the total transmit power of the user set is compose of transmit power of each user which transmit $R_{k, a v e} / M_{\tilde{n}, p}$ bits. Select the users set with the minimum transmit power in this way on each subcarrier.

In Step 4, If the assigned user $\hat{k}$ satisfies the required number of subcarriers, the rest of subcarriers will not be assigned to $\hat{k}$ user any more. As described in Subsection 3.1, once the $\hat{p}$ th user set is selected, the number of subcarriers for each user in the $\hat{p}$ th user set adds $1 / M_{\check{n}, p}$.
Algorithm 2. Simplified Normalized User Selection Algorithm (S-NUS)

In subcarrier assignment algorithm of NUS, Step 2 and Step 3 need to traverse all the candidate user sets on each subcarrier, it is complicated when the number of users is large. In order to further lower the complexity, S-NUS algorithm is proposed. Selecting a user with the largest channel gain, then select other users with large channel gain and low correlation with already selected users. In [6], it is shown that this algorithm can achieve the asymptotic performance as DPC with number of users increasing. The subcarrier assignment algorithm is shown as follows (Figure 1).

Step 1 is the same as NUS.

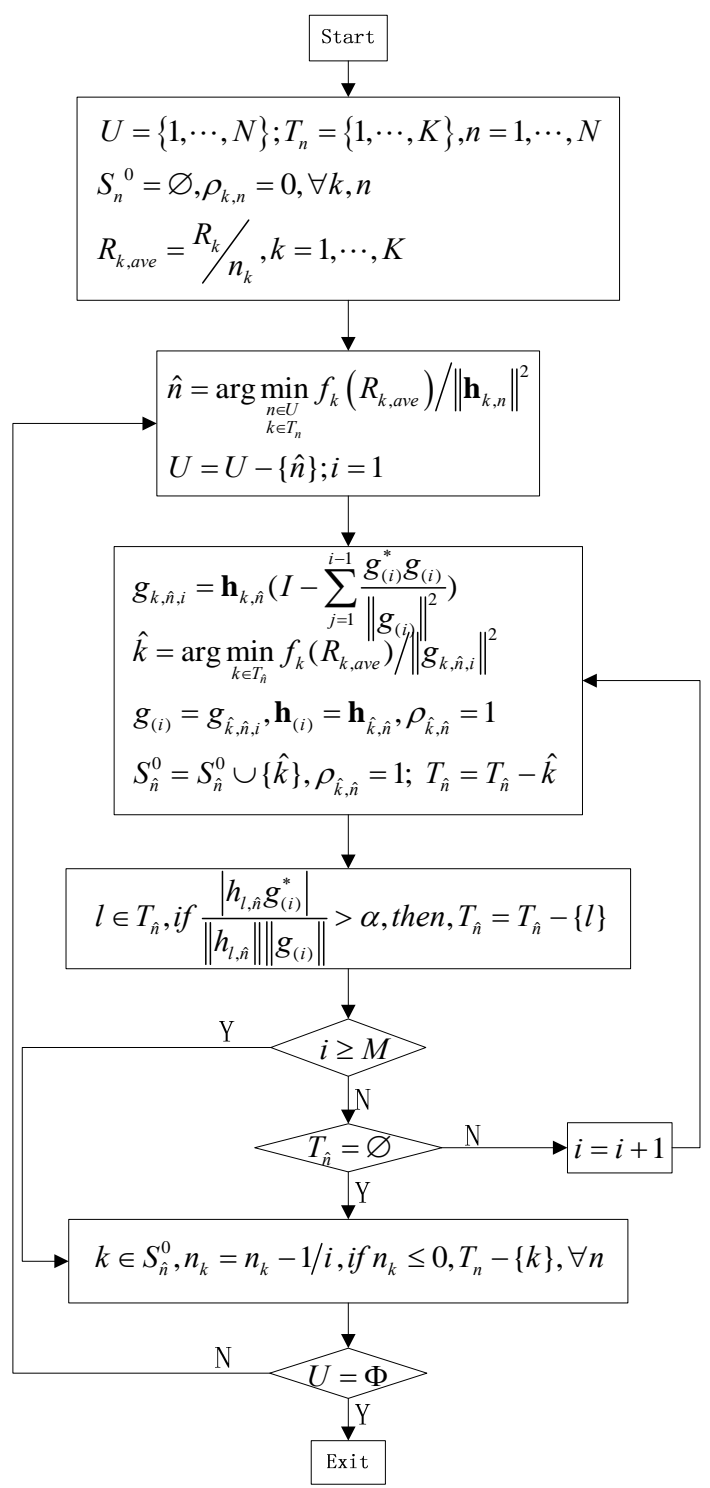

Figure 1. Flow chart of the subcarrier assignment algorithm. 


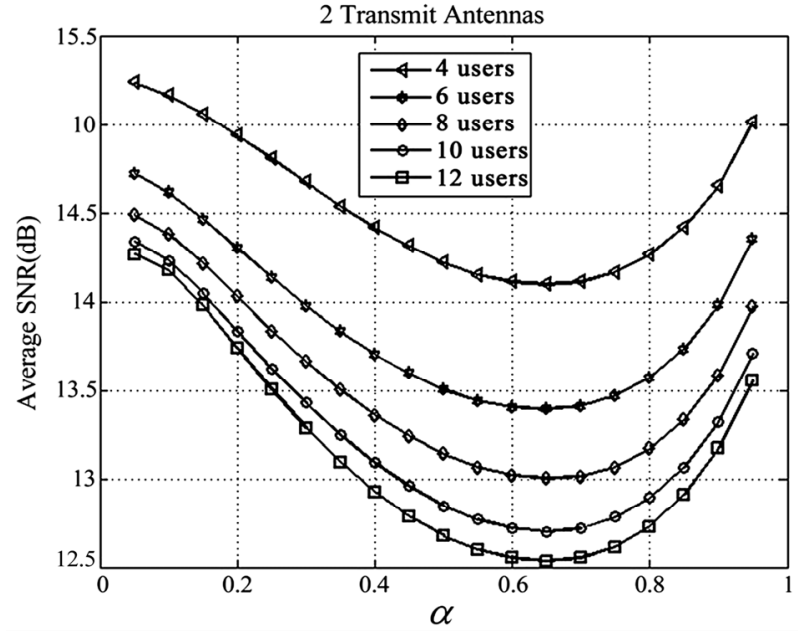

Figure 2. Required average SNR vs. different values of $\alpha$ with $\mathbf{M}=2$.

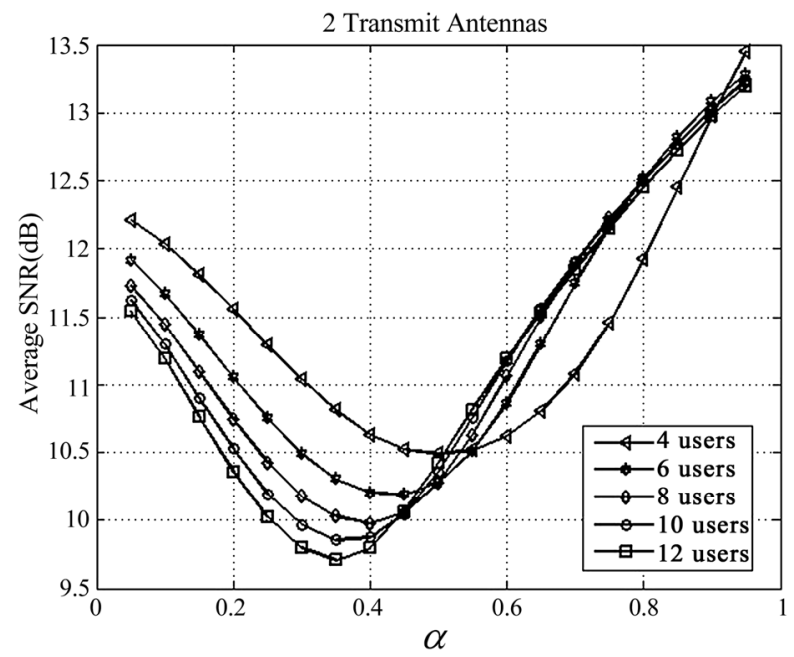

Figure 3. Required average SNR vs. different values of $\alpha$ with $\mathrm{M}=4$.

Step 2 Selecting the subcarrier is the same as NUS.

In Step 3, $g_{k, i}$ is the orthogonal component of $h_{k, n}$ spanned by

$$
\left\{g_{(1), n}, \ldots, g_{(i-1), n}\right\} \text {, }
$$

when $i=1$, this implies $g_{k, n, i}=\mathbf{h}_{k, n}$. [6] indicates that $g(i) \approx h(i)$ when the orthogonality of channels of users is good enough. The user $\hat{k}$ with the minimum transmit power is chosen while transmitting $R_{\hat{k} \text {,ave }}\left(\hat{k} \in T_{n}\right)$ bits every time.

In Step 4, if the remainder whose channels are not semi-orthogonal to the $\hat{k}$ th user's will be dropped off. $\alpha$ is a positive constant [6]. In ZFBF, selecting a non-orthogonal user degrades the effective chamnnel gain of the other users. Therefore, forcing semi-othogo- nal among users not only promotes the performance of the system but also reduces the complexity of the algorithm.

In Step 5, judge whether the number of subcarriers is satisfied and count the number of subcarriers as in NUS.

\subsection{Algorithmic Complexity}

In this section, the worst case performance of each algorithm is studied as a function of the number of transmission antennas $M$, the number of users $K$ and the number of subcarriers $N$. The optimal method for subcarriers allocation requires exhaustive search, so the computational complexity is

$$
O\left(\left(\begin{array}{l}
K \\
M
\end{array}\right)^{N}\right) .
$$

Computational complexity of the algorithm in [7] is $O\left(M^{2} K N^{2}\right)$. The NUS algorithm need to traverse all candidate user sets on each subcarrier, the process of traversing all the candidate user set needs

$$
O\left(M^{2}\left(\begin{array}{l}
K \\
M
\end{array}\right)\right)
$$

and selecting the subcarrier needs $O(N)$ on each subcarrier, so the computational complexity is

$O\left(M^{2} N^{2}\left(\begin{array}{l}K \\ M\end{array}\right)\right)$.

The S-NUS algorithm is similar to the algorithm in [7], but more simple, computational complexity is $O\left(M^{2} K N\right)$..

\section{Numerical Results}

Performance of the proposed algorithms is investigated in this section. An OFDM system with 128 subcarriers is considered. We assume that the channel of each antenna of each user is identically independent and experiences frequency selective fading. The sum target bit rate of users is $512 \mathrm{bits} / \mathrm{symbol}$ and target rate of each user is identical. For adaptive bit loading, QPSK, 16QAM, 64QAM and no data transmission are adopted here. When uncoded $2^{c}$-ary QAM is employed, the required average SNR can be tightly approximated as

$$
f_{k}(c)=\frac{\left(1-2^{c}\right) \log \left(5 B E R_{k}\right)}{1.6}[8] .
$$

Figure 2 shows that the required average SNR versus $\alpha$ when the number of transmission antennas is 2 . It is seen that the system achieves the best performance when the value of $\alpha$ is 0.65 . Figure 3 shows that the required average SNR versus $\alpha$ when the number of transmission antennas is 4 . It is seen that the system achieves the 
best performance when the value of $\alpha$ is between. $[0.35,0.50]$ The gap of performance is very big with different values of $\alpha$, so it is important to choose a suitable $\alpha$.Since the value of $\alpha$ in [7] is 1 , the performance is inferior to the proposed algorithms.

Figure 4 and Figure 5 show the performance of the proposed algorithms compared with the algorithm in [7] and none-adaptive algorithm when the number of transmission antennas is 2 and the number of users is 4 and 8 . Fig.6 and Figure 7 show the performance of the proposed algorithms compared with the algorithm in [7] and none-adaptive algorithm when the number of transmission antennas is 4 and the number of users is 4 and 8 . The value of $\alpha$ for S-NUS algorithm is 0.65 and 0.4 in two antenna configuration respectively. Each subcarrier is assigned to only one user in proper order in the none-adaptive method. From Figure 4 to Figure 7, it is seen that compared with the algorithm in [7], both $\mathrm{BABS}+\mathrm{NUS}$ and BABS+S-NUS achieve significant performance improvement.

Since the S-NUS method first selects a user with the minimum transmit power when transmitting $R_{k, \text { ave }}$ bits, then selects the users with large channel gain and low correlation with the other already selected users. But there is maybe a user set in which channel gain of users is not large enough but the orthogonality among users is better, this user set may be a better choice. NUS algorithm can select the better user set, hence, the performance of NUS is superior to S-NUS. But compared with NUS method, S-NUS method has only little performance loss with lower computational complexity, so S-NUS method is a better choice when the number of users is very large. Besides, because diversity of multiple users is applied, it is seen that the required average SNR is decreased with the increasing number of users.

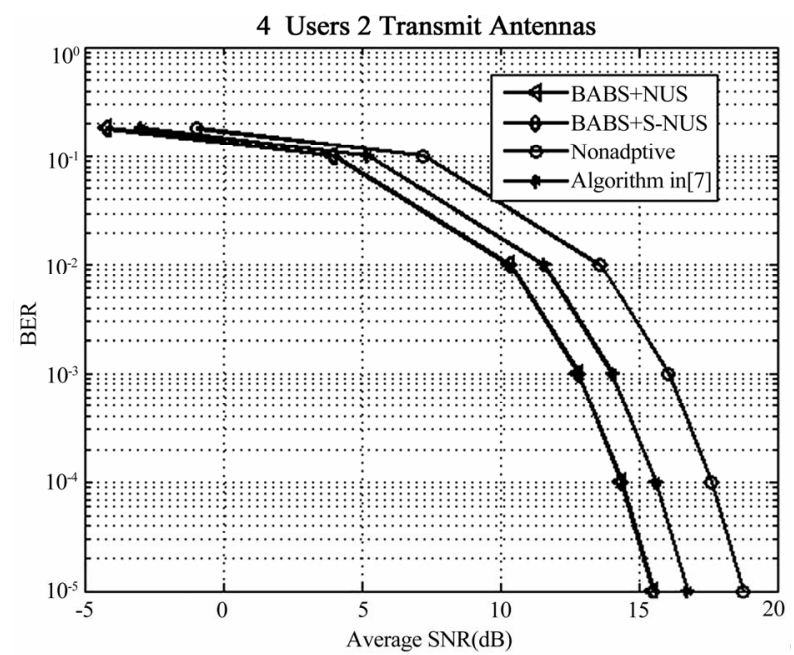

Figure 4. BER vs. required average SNR with $M=2$ when the number of user is 4 .

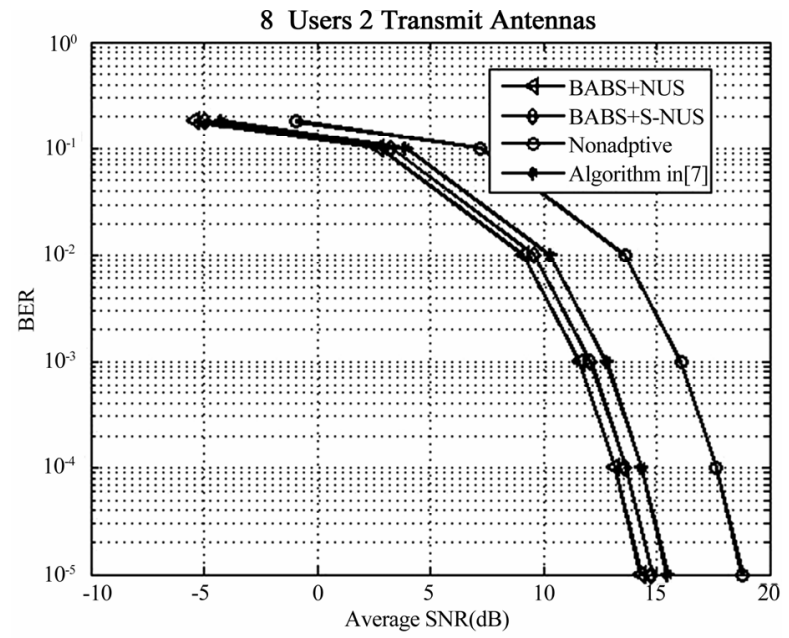

Figure 5. BER vs. required average SNR with $M=2$ when the number of user is 8 .

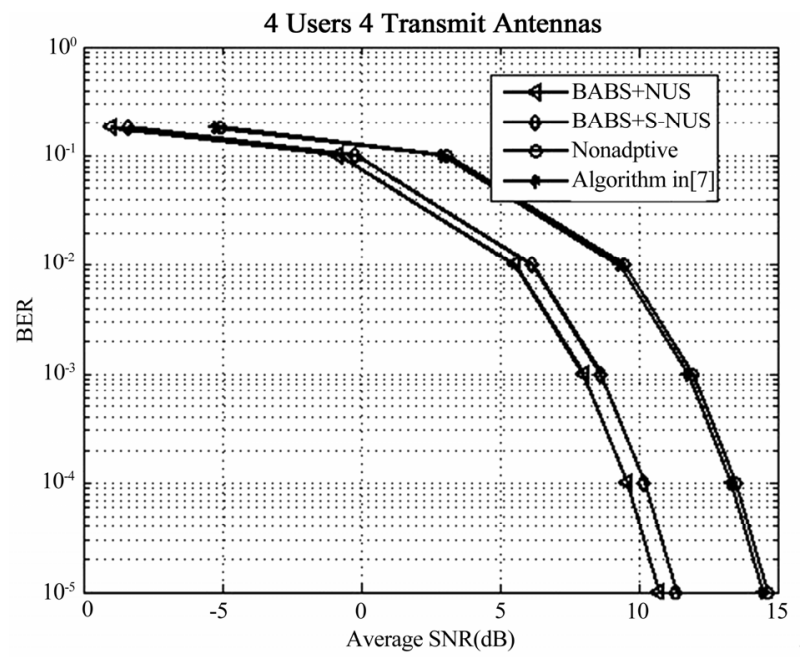

Figure 6. BER vs. required average SNR with $M=4$ when the number of user is 4 .

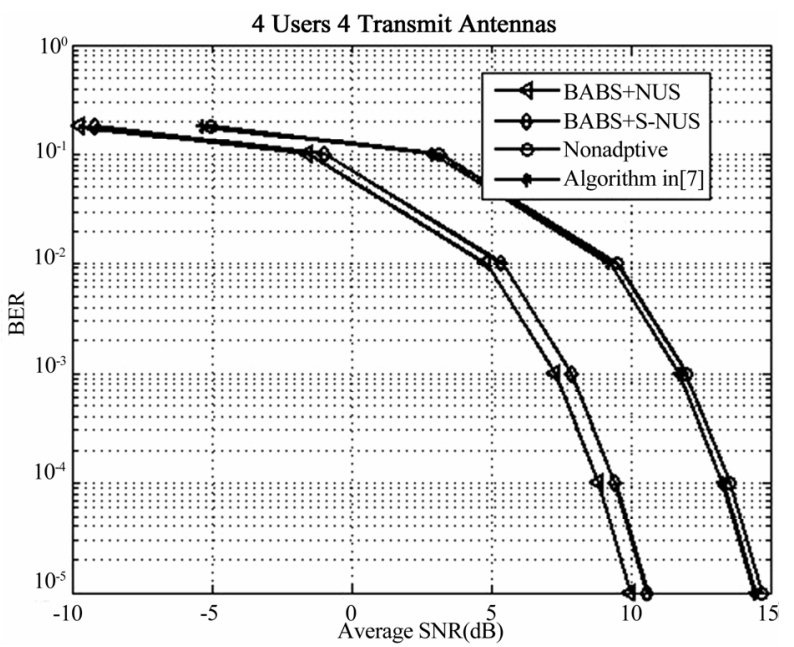

Figure 7. BER vs. required average SNR with $M=4$ when the number of user is 8 . 


\section{Conclusions}

Two suboptimal algorithms for subcarriers and power allocation among users in a MIMO-OFDM system have been described in this paper. Dividing the problem into two stages enabled the design of algorithms with low computational complexity, which operates well in our simulation. The NUS algorithm has a better performance but the complexity is larger, hence, the S-NUS algorithm has a good trade-off between the performance and the complexity. The numerical results show that both of the two proposed algorithms achieve better performance while the computational complexity is almost the same as the algorithm in [7]. Actually, if the resource allocation method for MIMO-OFDM systems is divided into two stages like this in this paper, the SDMA (Space-Division Multiple Access) grouping algorithm for MIMO systems can be employed. For example, the SUS (SemiOrthogonal User Selection) algorithm [6] is employed in the S-NUS algorithm. In next step, our research is to investigate more SDMA grouping algorithms and use them for the resource allocation in the MIMO-OFDM systems.

Besides, the ZFBF is power inefficient because beamforming weights are not matched to user channels. Therefore, the problem of resource allocation employing more efficient techniques such as MMSE-BF (Minimum Mean Square Error), RBF (Random Beamforming) need to be further explored.

\section{References}

[1] C. Y. Wong, R. S. Cheng, K. B. Letaief, and R. D. Murch, "Multiuser OFDM with adaptive subcarrier, bit, and power allocation,” IEEE Journal on Selected Areas in Communications, Vol. 17, pp. 1747-1758, October 1999.

[2] D. Kivanc, G. Q. Li, and H. Liu, "Computationally efficient bandwidth allocation and power control for OFDMA,” IEEE Transactions on Wireless Communications, Vol. 2, pp. 1150-1158, November 2003.

[3] I. Kim, I. S. Park, and Y. H. Lee, "Use of linear programming for dynamic subcarrier and bit allocation in multiuser OFDM," IEEE Transactions on Vehicular Technology, Vol. 55, pp. 1195-1207, July 2006.

[4] Y. M. Tsang and R. S. K. Cheng, "Optimal resource allocation in SDMA/multi-input-single-output/OFDM systems under QoS and power constraints,” in Proceedings of WCNC 2004, pp. 1595-1600, 2004.

[5] P. W. C. Chan and R. S. K. Cheng, "Reduced-complexity power allocation in zero-forcing MIMO-OFDM downlink system with multiuser diversity," in Proceedings of ISIT 2005, pp. 2320-2324, 2005.

[6] T. Yoo and A. Goldsmith, “On the optimality of multiantenna broadcast scheduling using zero-forcing beamforming," IEEE Journal on Selected Areas in Communications, Vol. 24, pp. 528-541, March 2006.

[7] Y. Shin, T. S. Kang, and H. M. Kin, “An efficient resource allocation for multiuser MIMO-OFDM systems with zero-forcing beamformer," in Proceedings of PIMRC 2007, pp. 1-5, 2007.

[8] S. T. Chung and A. J. Goldsmith, "Degree of freedom in adaptive modulation: A unified view,” IEEE Transactions on Communications, Vol. 49, pp. 1561-1571, September, 2001. 